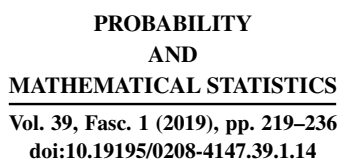

\title{
STOCHASTIC COMPLEX INTEGRALS ASSOCIATED WITH HOMOGENEOUS INDEPENDENTLY SCATTERED RANDOM MEASURES ON THE LINE
}

BY

\author{
KOUJI YAMAMURO (GIFU)
}

\begin{abstract}
Complex integrals associated with homogeneous independently scattered random measures on the line are discussed. Theorems corresponding to Cauchy's theorem and the residue theorem are given. Furthermore, the converse of Cauchy's theorem is discussed.
\end{abstract}

2010 AMS Mathematics Subject Classification: Primary: 60E07; Secondary: $60 \mathrm{H} 05$.

Key words and phrases: Stochastic integral, infinitely divisible distribution, Lévy process, complex integral.

\section{INTRODUCTION}

A Lévy process is a stochastic process with stationary independent increments, stochastically continuous, starting at the origin, and having càdlàg paths. If the stationarity of increments is not assumed, it is called an additive process. Our notation and definition follow [15]. Let $\left\{X_{t}: t \geqslant 0\right\}$ be a Lévy process on $\mathbb{R}$. The distribution of $X_{1}$ has the Lévy-Khintchine representation of the form

$$
\begin{aligned}
E e^{i \xi X_{1}} & =\exp [C(\xi)], \\
C(\xi) & =-2^{-1} A \xi^{2}+i \gamma \xi+\int_{\mathbb{R}}\left(e^{i \xi x}-1-i \frac{\xi x}{1+x^{2}}\right) \nu(d x),
\end{aligned}
$$

where $A \geqslant 0, \gamma \in \mathbb{R}, \nu(\{0\})=0$ and $\int_{\mathbb{R}} \min \left\{1,|x|^{2}\right\} \nu(d x)<\infty$. Here $C(\xi)$ is the cumulant function of the distribution of $X_{1}$. Stochastic integrals of nonrandom functions with repect to additive processes on $\mathbb{R}$ were studied by [4], [12] and [24]. Ken-iti Sato developed this theory in [116] and [117]. He has carried out various studies in [18]-[22]. These days, the connections between stochastic integral mappings and subclasses of infinitely divisible distributions are found. See [1]], [2], [5], [7], [8] and [23]. Our aim is to study stochastic complex integrals based on the theory developed by Sato. Let $z=s+i t \in \mathbb{C}$, where $s$ and $t$ are 
the real and the imaginary part of $z$, respectively. A usual complex integral is an integral with respect to $d z=d s+i d t$. Our complex integral is an integral with respect to $d z_{\mathbf{X}}=d s+i d X_{t}$. Here we consider a process $\mathbf{X}=\left\{X_{t}: t \in \mathbb{R}\right\}$ on $\mathbb{R}$ defined along the construction in [13] as follows. Let $\left\{X_{t}^{(1)}: t \geqslant 0\right\}$ and $\left\{X_{t}^{(2)}: t \geqslant 0\right\}$ be independent Lévy processes such that $E e^{i \xi X_{t}^{(1)}}=\exp [t C(\xi)]$ and $E e^{i \xi X_{t}^{(2)}}=\exp [t C(-\xi)]$. The process $\mathbf{X}$ is defined by

$$
X_{t}= \begin{cases}X_{t}^{(1)} & \text { for } t \geqslant 0 \\ X_{(-t)-}^{(2)} & \text { for } t<0\end{cases}
$$

Throughout this paper, let $\mathbf{X}=\left\{X_{t}: t \in \mathbb{R}\right\}$ be the above process over an infinite time parameter set $\mathbb{R}$. We call $(A, \nu, \gamma)$ the generating triplet of $\mathbf{X}$.

Here we mention some notation and definitions: $\lambda$ stands for the Lebesgue measure on $\mathbb{R}$. Let $J$ be an interval in $\mathbb{R}$, for example, $J=\mathbb{R},(-\infty, 0)$ or $[0, \infty)$. $\mathcal{B}_{J}^{0}$ is the class of bounded Borel sets in $J$; p-lim stands for limit in probability; $\partial B$ stands for the boundary of $B$ and we set $\bar{B}=B \cup \partial B$ for any set $B$ in $\mathbb{R}^{2}$. By a curve $\Gamma$ we mean a function $\Gamma:[0,1] \rightarrow \mathbb{R}^{2}$ which is of class $C^{1}$. We call $\Gamma(0)$ and $\Gamma(1)$ the beginning point and the end point of $\Gamma$, respectively. By a path $\Gamma$ we mean a sequence of curves, $\Gamma=\left\{\Gamma_{1}, \Gamma_{2}, \ldots, \Gamma_{n}\right\}$ such that the end point of $\Gamma_{j}$ is the beginning point of $\Gamma_{j+1}$ for $j=1,2, \ldots, n-1$. A path $\Gamma$ is called a closed path if the end point of $\Gamma_{n}$ is the beginning point of $\Gamma_{1}$. If a closed path $\Gamma$ does not intersect itself, we call it a simple closed path. A path $\Gamma$ is called regular if $\Gamma_{j}^{\prime} \neq 0$ for each $j$.

In this paper, let $D$ be a bounded, connected and open set in $\mathbb{R}^{2}$, and suppose that $\partial D=\bigcup_{j=0}^{q} \Gamma^{j}$, where $0 \leqslant q<\infty$ and each $\Gamma^{j}$ is a regular, simple closed path. In the case $q \geqslant 1$, we suppose that the region surrounded by $\Gamma^{0}$ includes $\bigcup_{j=1}^{q} \Gamma^{j}$, and that $\Gamma^{0}$ is parameterized counterclockwise and $\Gamma^{j}, j=1,2, \ldots, q$, are parameterized clockwise. Let $\bar{D} \subset \widetilde{D} \subset[a, b] \times[c, d]$ for some open set $\widetilde{D}$ and some $a, b, c, d \in \mathbb{R}$. Let $f(s, t)$ be an $\mathbb{R}$-valued measurable function on $[a, b] \times$ $[c, d]$ and of class $C^{1}$ on $\widetilde{D}$. We identify $z=s+i t \in \mathbb{C}$ with $(s, t) \in \mathbb{R}^{2}$. Then $f(z)$ is regarded as a function of $(s, t)$ and sometimes denoted by $f(s, t)$. Likewise, $[a, b] \times[c, d]$ is identified with $\{s+i t:(s, t) \in[a, b] \times[c, d]\}$. Now we define stochastic line integrals along a simple closed path $\Gamma$. Let

$$
\Delta_{s}^{n}=\left\{\left[s_{i-1}^{n}, s_{i}^{n}\right]: i=1,2, \ldots, m_{n}\right\} \quad \text { and }{ }_{t}^{n} \Delta=\left\{\left[t_{j-1}^{n}, t_{j}^{n}\right]: j=1,2, \ldots, l_{n}\right\}
$$

be partitions of $[a, b]$ and $[c, d]$, respectively. Here $s_{0}^{n}=a, s_{m_{n}}^{n}=b, t_{0}^{n}=c$, and $t_{l_{n}}^{n}=d$. The set of rectangles

$$
\Delta_{n}=\left\{I_{i, j}^{n}:=\left[s_{i-1}^{n}, s_{i}^{n}\right] \times\left[t_{j-1}^{n}, t_{j}^{n}\right]: i=1,2, \ldots, m_{n}, j=1,2, \ldots, l_{n}\right\}
$$

is called a partition of $[a, b] \times[c, d]$. Denote by $\left|I_{i, j}^{n}\right|$ the length of the diagonal of $I_{i, j}^{n}$. Then the size of $\Delta_{n}$ is defined by

$$
\left|\Delta_{n}\right|=\max \left\{\left|I_{i, j}^{n}\right|: i=1,2, \ldots, m_{n}, j=1,2, \ldots, l_{n}\right\} .
$$


Let $I_{i, j}^{n} \subset \widetilde{D}$. If $\partial I_{i, j}^{n}$ is parameterized counterclockwise, we define the line integral along $\partial I_{i, j}^{n}$ as follows:

$$
\begin{aligned}
\int_{\partial I_{i, j}^{n}} f(s, t) d X_{t} & =\int_{t_{j-1}^{n}}^{t_{j}^{n}} f\left(s_{i}^{n}, t\right) d X_{t}-\int_{t_{j-1}^{n}}^{t_{j}^{n}} f\left(s_{i-1}^{n}, t\right) d X_{t} \\
& =\int_{t_{j-1}^{n}}^{t_{j}^{n}}\left(\int_{s_{i-1}^{n}}^{s_{i}^{n}} \frac{\partial f}{\partial s}(s, t) d s\right) d X_{t} .
\end{aligned}
$$

Here we interpret the values of the line integrals along $\left[s_{i-1}^{n}, s_{i}^{n}\right] \times\left\{t_{j}\right\}$ and $\left[s_{i-1}^{n}, s_{i}^{n}\right] \times\left\{t_{j-1}\right\}$ as zero. Hence we are able to introduce the following definition of the line integral along $\partial D$. The way of the definition is the first key to our analysis.

DEFINITION 1.1. Let $D_{n}=\bigcup_{I_{i, j}^{n} \subset \bar{D}} I_{i, j}^{n}$ and $D^{n}=\bigcup_{I_{i, j}^{n} \cap \bar{D} \neq \emptyset} I_{i, j}^{n}$, where $D^{n} \subset \widetilde{D}$. Then $D_{n}$ and $D^{n}$ are called the inner partition and the outer partition of $\bar{D}$ with respect to $\Delta_{n}$, respectively. For each $j$, we set

$$
\begin{aligned}
& D_{n, j}=\left\{s:(s, t) \in D_{n}, t \in\left(t_{j-1}^{n}, t_{j}^{n}\right)\right\}, \\
& D^{n, j}=\left\{s:(s, t) \in D^{n}, t \in\left(t_{j-1}^{n}, t_{j}^{n}\right)\right\} .
\end{aligned}
$$

Let $f(s, t)$ be of class $C^{1}$ on $\widetilde{D}$. If

$$
\int_{\partial D_{n}} f(s, t) d X_{t}=\sum_{I_{i, j}^{n} \subset \bar{D}} \int_{\partial I_{i, j}^{n}} f(s, t) d X_{t}=\sum_{j=1}^{l_{n}} \int_{t_{j-1}^{n}}^{t_{j}^{n}}\left(\int_{D_{n, j}} \frac{\partial f}{\partial s}(s, t) d s\right) d X_{t}
$$

and

$$
\int_{\partial D^{n}} f(s, t) d X_{t}=\sum_{I_{i, j}^{n} \cap \bar{D} \neq \emptyset} \int_{\partial I_{i, j}^{n}} f(s, t) d X_{t}=\sum_{j=1}^{l_{n}} \int_{t_{j-1}^{n}}^{t_{j}^{n}}\left(\int_{D^{n, j}} \frac{\partial f}{\partial s}(s, t) d s\right) d X_{t}
$$

converge in probability and these limits are equal almost surely as $\left|\Delta_{n}\right| \rightarrow 0$ for any sequence of partitions $\Delta_{n}$ and if the limit does not depend on the choice of the sequence $\left\{\Delta_{n}\right\}$, then we call this limit the stochastic line integral of $f$ along $\partial D$, and denote it by

$$
\int_{\partial D} f(s, t) d X_{t} .
$$

REMARK 1.1. (i) We note that $D_{n} \subset \bar{D} \subset D^{n} \subset \widetilde{D}$. We appropriate the path $\partial D$ by the boundaries $\partial D_{n}$ and $\partial D^{n}$.

(ii) If $D_{n, j}=\emptyset$, then we interpret the value of $\int_{D_{n, j}} \frac{\partial f}{\partial s}(s, t) d s$ as zero. Likewise, $\int_{D^{n, j}} \frac{\partial f}{\partial s}(s, t) d s=0$ if $D^{n, j}=\emptyset$. 
In Section 2, stochastic integrals with respect to $\mathbf{X}$ are introduced and useful propositions are given without proofs. Section 3 begins with Green's theorem and Cauchy's theorem and the residue theorem are given. Furthermore, the converse of Cauchy's theorem is investigated. Proofs of the assertions in Section 3 are given in Section 4.

\section{STOCHASTIC INTEGRALS}

We show first the relationship of the process $\mathbf{X}=\left\{X_{t}: t \in \mathbb{R}\right\}$ to an independently scattered random measure (i.s.r.m.) on $\mathbb{R}$, and define stochastic integrals with respect to $\mathbf{X}$. Following [20], we define a homogeneous i.s.r.m. over $\mathbb{R}$. Also refer to [6], [12], [14], [16] and [24]. Here we note that A. Prékopa studied independently scattered random measures (not necessarily homogeneous) in the series of papers [9]-[II] . In this section, propositions are given without proofs.

DEFINITION 2.1. A family of $\mathbb{R}$-valued random variables $\left\{X(B): B \in \mathcal{B}_{\mathbb{R}}^{0}\right\}$ is called an independently scattered random measure (i.s.r.m.) if it satisfies the following conditions:

(i) $\sum_{n=1}^{\infty} X\left(B_{n}\right)$ converges a.s. and equals $X\left(\sum_{n=1}^{\infty} B_{n}\right)$ a.s. for any sequence $B_{1}, B_{2}, \ldots$ of disjoint sets in $\mathcal{B}_{\mathbb{R}}^{0}$ with $\sum_{n=1}^{\infty} B_{n} \in \mathcal{B}_{\mathbb{R}}^{0}$;

(ii) $X\left(B_{1}\right), \ldots, X\left(B_{n}\right)$ are independent for any finite sequence $B_{1}, B_{2}, \ldots$, $B_{n}$ of disjoint sets in $\mathcal{B}_{\mathbb{R}}^{0}$;

(iii) $X(\{a\})=0$ a.s. for every $a \in \mathbb{R}$.

In addition, $\{X(B)\}$ is called a homogeneous i.s.r.m. if it satisfies

(iv) $X(B) \stackrel{d}{=} X(B+a)$ for every $B \in \mathcal{B}_{\mathbb{R}}^{0}$ and $a \in \mathbb{R}$.

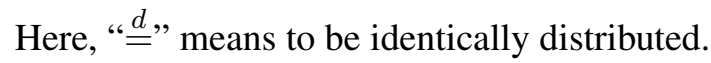

We give a proposition corresponding to Theorem 3.2 (ii) of [16]. The proof is similar to that of Theorem 3.2 of [16].

Proposition 2.1. There is a unique homogeneous i.s.r.m. $\left\{X(B): B \in \mathcal{B}_{\mathbb{R}}^{0}\right\}$ such that

$$
X_{t}-X_{s}=X((s, t]) \quad \text { for } s \text { and } t \text { with } s<t \text {. }
$$

Further, we have

$$
E e^{i \xi X(B)}=\exp [\lambda(B) C(\xi)]
$$

In particular, we have

$$
X(B)= \begin{cases}X^{(1)}(B) \text { a.s. } & \text { for } B \in \mathcal{B}_{[0, \infty)}^{0}, \\ -X^{(2)}(-B) \text { a.s. } & \text { for } B \in \mathcal{B}_{(-\infty, 0)}^{0}\end{cases}
$$


Here, independently scattered random measures $X^{(1)}$ and $X^{(2)}$ correspond to $X_{t}^{(1)}$ and $X_{t}^{(2)}$, respectively.

REMARK 2.1. The symbol " $-B$ " means the set $\{-x: x \in B\}$. Uniqueness means that if both $\{X(B)\}$ and $\left\{X^{\prime}(B)\right\}$ satisfy (2.1), then $X(B)=X^{\prime}(B)$ a.s. for every $B \in \mathcal{B}_{\mathbb{R}}^{0}$.

Although Rocha-Arteaga and Sato [13] directly define stochastic integrals based on $\left\{X_{t}: t \in \mathbb{R}\right\}$, we define those integrals based on independently scattered random measures.

DEFINITION 2.2. An $\mathbb{R}$-valued function $F(t)$ on $\mathbb{R}$ is called a simple function if

$$
F(t)=\sum_{j=1}^{n} 1_{B_{j}}(t) R_{j}
$$

for some $n$, where $B_{1}, \ldots, B_{n}$ are disjoint Borel sets in $\mathbb{R}$ and $R_{1}, \ldots, R_{n} \in \mathbb{R}$. If $F$ is a simple function of this form, then we define the integral of $F$ over $B \in \mathcal{B}_{\mathbb{R}}^{0}$ with respect to $\mathbf{X}$ as

$$
\int_{B} F(t) d X_{t}=\sum_{j=1}^{n} R_{j} X\left(B \cap B_{j}\right) .
$$

Definition 2.3. An $\mathbb{R}$-valued function $F(t)$ on $\mathbb{R}$ is said to be locally $\mathbf{X}$ integrable or locally $\left\{X_{t}\right\}$-integrable if it is measurable and there is a sequence of simple functions $F_{n}(t), n=1,2, \ldots$, such that

(1) $F_{n}(t) \rightarrow F(t)$ a.e. as $n \rightarrow \infty$, and

(2) for every $B \in \mathcal{B}_{\mathbb{R}}^{0}, \int_{B} F_{n}(t) d X_{t}$ is convergent in probability as $n \rightarrow \infty$.

We denote by $\mathbf{L}(\mathbf{X})$ the class of locally $\mathbf{X}$-integrable functions.

Proposition 2.15 of [17] remains true even if we replace $\mathcal{B}_{[0, \infty)}^{0}$ with $\mathcal{B}_{\mathbb{R}}^{0}$ :

Proposition 2.2. Let $F \in \mathbf{L}(\mathbf{X})$. If $F_{n}^{1}(t)$ and $F_{n}^{2}(t)$ are sequences satisfying (1) and (2) of Definition 2.3, then

$$
\text { p- } \lim _{n \rightarrow \infty} \int_{B} F_{n}^{1}(t) d X_{t}=\mathrm{p}-\lim _{n \rightarrow \infty} \int_{B} F_{n}^{2}(t) d X_{t} \text { a.s. for each } B \in \mathcal{B}_{\mathbb{R}}^{0} .
$$

Proposition 2.2 enables us to define stochastic integrals of locally $\mathbf{X}$-integrable functions:

Definition 2.4. Let $F \in \mathbf{L}(\mathbf{X})$ and let simple functions $F_{n}, n=1,2, \ldots$, satisfy (1) and (2) of Definition 2.3. Then we define

$$
\int_{B} F(t) d X_{t}=\mathrm{p}-\lim _{n \rightarrow \infty} \int_{B} F_{n}(t) d X_{t} \quad \text { for } B \in \mathcal{B}_{\mathbb{R}}^{0} .
$$


Prékopa's study gives us useful propositions, see Theorems 2.1, 2.4 and 2.5 of [10].

Proposition 2.3. If $F_{1}, F_{2} \in \mathbf{L}(\mathbf{X})$, then, for any $a_{1}, a_{2} \in \mathbb{R}, a_{1} F_{1}+a_{2} F_{2} \in$ $\mathbf{L}(\mathbf{X})$ and

$$
\int_{B}\left(a_{1} F_{1}(t)+a_{2} F_{2}(t)\right) d X_{t}=a_{1} \int_{B} F_{1}(t) d X_{t}+a_{2} \int_{B} F_{2}(t) d X_{t} \quad \text { a.s. }
$$

for $B \in \mathcal{B}_{\mathbb{R}}^{0}$.

Proposition 2.4. Let $F \in \mathbf{L}(\mathbf{X})$ and let $B_{1}, B_{2} \in \mathcal{B}_{\mathbb{R}}^{0}$. If the intersection of $B_{1}$ and $B_{2}$ is empty, or if it consists of a finite number of points, then

$$
\int_{B_{1} \cup B_{2}} F(t) d X_{t}=\int_{B_{1}} F(t) d X_{t}+\int_{B_{2}} F(t) d X_{t} \quad \text { a.s. }
$$

Local integrability is characterized in terms of the generating triplet $(A, \nu, \gamma)$ of $\mathbf{X}$, see Theorem 2.7 of [12]. Now we define

$$
\begin{aligned}
\varphi(u)= & A u^{2}+\int_{\mathbb{R}} \min \left\{1,|u x|^{2}\right\} \nu(d x) \\
& +\left|u \gamma+\int_{\mathbb{R}}\left(\frac{u x}{1+|u x|^{2}}-\frac{u x}{1+|x|^{2}}\right) \nu(d x)\right|
\end{aligned}
$$

for $u \in \mathbb{R}$.

PROPOSITION 2.5. Let $F(t)$ be an $\mathbb{R}$-valued measurable function on $\mathbb{R}$. Then $F \in \mathbf{L}(\mathbf{X})$ if and only if

$$
\int_{t_{0}}^{t_{1}} \varphi(F(t)) d t<\infty \quad \text { for }-\infty<t_{0}<t_{1}<\infty
$$

COROLLARY 2.1. Let $F(t)$ be an $\mathbb{R}$-valued measurable function on $\mathbb{R}$. If $F(t)$ is locally bounded, then $F \in \mathbf{L}(\mathbf{X})$.

Lastly, we give an expression of the characteristic function of $\int_{B} F(t) d X_{t}$. See Proposition 2.6 of [II2].

Proposition 2.6. If $F \in \mathbf{L}(\mathbf{X})$, then

$$
E \exp \left[i \xi \int_{B} F(t) d X_{t}\right]=\exp \left[\int_{B} C(F(t) \xi) d t\right]
$$

for $B \in \mathcal{B}_{\mathbb{R}}^{0}$. 


\section{STOCHASTIC COMPLEX INTEGRALS}

First, we define double integrals with repect to $d s d X_{t}$.

Definition 3.1. Let $c \leqslant t \leqslant d$ and set $B_{t}=\{s:(s, t) \in B \cap \widetilde{D}\}$ for every Borel set $B \subset[a, b] \times[c, d]$. Suppose $f(s, t)$ is an $\mathbb{R}$-valued function of class $C^{1}$ on $\widetilde{D}$. Then we define

$$
\iint_{B} f(s, t) d s d X_{t}=\int_{c}^{d}\left(\int_{B_{t}} \frac{\partial f}{\partial s}(s, t) d s\right) d X_{t}
$$

Here, if $B_{t}=\emptyset$, then we interpret the value of $\int_{B_{t}} \frac{\partial f}{\partial s}(s, t) d s$ as zero.

As the second key of our analysis, we will show a theorem corresponding to Green's theorem. Recall that $\partial D=\bigcup_{j=0}^{q} \Gamma^{j}$, where $\Gamma^{0}$ is parameterized counterclockwise and $\Gamma^{j}, j=1,2, \ldots, q$, are parameterized clockwise.

THEOREM 3.1. Suppose $f(s, t)$ is an $\mathbb{R}$-valued function of class $C^{1}$ on $\widetilde{D}$. Then

$$
\int_{\partial D} f(s, t) d X_{t}=\iint_{D} \frac{\partial f}{\partial s}(s, t) d s d X_{t} \quad \text { a.s. }
$$

REMARK 3.1. The function $\int_{(\bar{D})_{t}} \frac{\partial f}{\partial s}(s, t) d s$ is locally $\mathbf{X}$-integrable because it is bounded and we can use Corollary 2.1. Hence the right-hand side of (B.D) is definable.

Let $f(z)$ be a complex-valued function for $z=s+i t \in \widetilde{D}$. Then $f(z)$ can be expressed as $f(z)=u(z)+i v(z)$, where $u(z)$ and $v(z)$ are $\mathbb{R}$-valued functions.

Definition 3.2. Suppose $\int_{\partial D} u(s, t) d X_{t}$ and $\int_{\partial D} v(s, t) d X_{t}$ are definable. Then we define stochastic complex integrals along a closed path $\partial D$ as follows:

$$
\begin{aligned}
\int_{\partial D} f(z) d z_{\mathbf{X}} & =\int_{\partial D}(u(s, t)+i v(s, t))\left(d s+i d X_{t}\right) \\
& =\int_{\partial D} u(s, t) d s-\int_{\partial D} v(s, t) d X_{t}+i\left(\int_{\partial D} v(s, t) d s+\int_{\partial D} u(s, t) d X_{t}\right) .
\end{aligned}
$$

From Theorem 3.1 we obtain a theorem corresponding to Cauchy's theorem:

THEOREM 3.2. Suppose $f(z)$ is holomorphic on $\widetilde{D}$. Then

$$
\int_{\partial D} f(z) d z_{\mathbf{X}}=i \iint_{\bar{D}} \frac{\partial f}{\partial s}(s, t) d s d\left(X_{t}-t\right) \text { a.s. }
$$

REMARK 3.2. (i) The process $\left\{X_{t}-t: t \in \mathbb{R}\right\}$ corresponds to a homogeneous i.s.r.m. $\left\{X(B)-\lambda(B): B \in \mathcal{B}_{\mathbb{R}}^{0}\right\}$. See Lemma 4.2 in Section 4 below. 
(ii) If $f(z)$ is holomorphic, then $u(s, t)$ and $v(s, t)$ are of class $C^{1}$.

(iii) In the case $X_{t}=t$, the stochastic complex integral is understood as the usual complex integral. Then the right-hand side of (3.2) is equal to zero, and the assertion coincides with Cauchy's theorem.

Let $f(s, t)$ be of class $C^{1}$ on $\widetilde{D}$ excluding a finite number of points. In particular, we suppose $D$ is simply connected. As $\int_{\partial D} f(s, t) d X_{t}$ is not definable in the sense of Definition 1.1, we define stochastic line integrals as follows:

DEFINITION 3.3. Let $D$ be a bounded, simply connected, open set and let $f(s, t)$ be an $\mathbb{R}$-valued function of class $C^{1}$ on $\widetilde{D} \backslash\left\{a_{1}, a_{2}, \ldots, a_{n}\right\}$, where $a_{j}=$ $\left(a_{j}^{1}, a_{j}^{2}\right) \in D$ for $j=1,2, \ldots, n$. Let

$$
I_{\epsilon, \delta}\left(a_{j}\right)=\left[a_{j}^{1}-\epsilon, a_{j}^{1}+\epsilon\right] \times\left[a_{j}^{2}-\delta, a_{j}^{2}+\delta\right],
$$

where $\partial I_{\epsilon, \delta}\left(a_{j}\right)$ is parameterized counterclockwise, and let

$$
D_{\epsilon, \delta}=D \backslash \bigcup_{j=1}^{n} I_{\epsilon, \delta}\left(a_{j}\right)
$$

Here we take $\epsilon$ and $\delta$ such that $I_{\epsilon, \delta}\left(a_{j}\right) \subset D$ and that $I_{\epsilon, \delta}\left(a_{j}\right), j=1,2, \ldots, n$, are disjoint. Then we define

$$
\int_{\partial D} f(z) d z_{\mathbf{X}}=\mathrm{p}-\lim _{\delta \downarrow 0}\left(\int_{\partial D_{\epsilon, \delta}} f(z) d z_{\mathbf{X}}+\sum_{j=1}^{n} \int_{\partial I_{\epsilon, \delta}\left(a_{j}\right)} f(z) d z_{\mathbf{X}}\right)
$$

and

$$
\int_{\partial D} f(s, t) d X_{t}=\mathrm{p}-\lim _{\delta \downarrow 0}\left(\int_{\partial D_{\epsilon, \delta}} f(s, t) d X_{t}+\sum_{j=1}^{n} \int_{\partial I_{\epsilon, \delta}\left(a_{j}\right)} f(s, t) d X_{t}\right),
$$

if each convergence on the right-hand sides does not depend on $\epsilon$. Here the integral

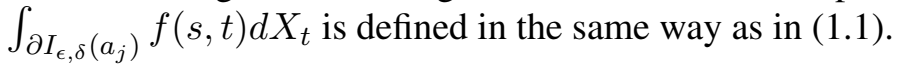

REMARK 3.3. If we regard $\partial I_{\epsilon, \delta}\left(a_{j}\right)$ as a part of $\partial D_{\epsilon, \delta}$, then $\partial I_{\epsilon, \delta}\left(a_{j}\right)$ is parameterized clockwise.

In Theorem 3.3 and Corollary 3.1 below, we suppose $D$ is simply connected.

TheOREM 3.3. Let $D$ be a bounded, simply connected, open set. Suppose $f(z)$ is holomorphic on $\widetilde{D}$ except at a finite number of isolated singular points $a_{1}, a_{2}, \ldots, a_{n} \in D$. Then we have the following representation:

$$
\int_{\partial D} f(s, t) d\left(X_{t}-t\right)=\mathrm{p}-\lim _{\delta \downarrow 0} \int_{\partial D_{\epsilon, \delta}} f(s, t) d\left(X_{t}-t\right)
$$


and

$$
\int_{\partial D} f(z) d z_{\mathbf{X}}=2 \pi i \sum_{j=1}^{n} \operatorname{Res}\left(f, a_{j}\right)+i \int_{\partial D} f(s, t) d\left(X_{t}-t\right) \quad \text { a.s. }
$$

Here, $\operatorname{Res}\left(f, a_{j}\right)$ stands for the residue of $f(z)$ at $a_{j}$ for $j=1,2, \ldots, n$.

REMARK 3.4. We note that the limit $\mathrm{p}-\lim _{\delta \downarrow 0} \int_{D_{\epsilon, \delta}} f(s, t) d\left(X_{t}-t\right)$ of (3.3) does not depend on $\epsilon$.

Here we mention the case where the number of isolated singular points is equal to one without proof. It is immediately derived from Theorem [3.3.

Corollary 3.1. Suppose $f(z)$ is holomorphic on $\widetilde{D}$. For $\eta \in D$, we have

$$
\int_{\partial D} \frac{f(z)}{z-\eta} d z_{\mathbf{X}}=2 \pi i f(\eta)+i \int_{\partial D} \frac{f(z)}{z-\eta} d\left(X_{t}-t\right) \quad \text { a.s. }
$$

REMARK 3.5. We do not state particularly that $f(\eta)$ has a similar representation to Cauchy's integral formula because $f(\eta)$ is non-random and the integrals are random, excluding the case $X_{t}=t$.

At the end of this section, we investigate the converse of Cauchy's theorem. We obtain the following fact:

TheOREM 3.4. Suppose that $E\left|X_{1}\right|<\infty$ and $E X_{1}=1$. Then, for any $\mathbb{R}$ valued function $f(s, t)$ of class $C^{1}$ on $\widetilde{D}$,

$$
E\left|\int_{\partial D} f(z) d z_{\mathbf{X}}\right|<\infty \text { and } E\left[\int_{\partial D} f(z) d z_{\mathbf{X}}\right]=\int_{\partial D} f(z) d z .
$$

Corollary 3.2. Suppose that $E\left|X_{1}\right|<\infty$ and $E\left[X_{1}\right]=1$. Let $u(s, t)$ and $v(s, t)$ be $\mathbb{R}$-valued functions of class $C^{1}$ on $\widetilde{D}$. Then $f(z)$ is holomorphic on $\widetilde{D}$ if and only if $E\left[\int_{\partial \triangle} f(z) d z_{\mathbf{X}}\right]=0$ for every closed triangle $\triangle \subset \widetilde{D}$.

REMARK 3.6. (i) The symbol $\triangle$ is used instead of $D$ because the paths of stochastic integrals are triangular paths.

(ii) If $X_{t}=t$, then the corollary states the assertion of Morera's theorem.

\section{PROOFS}

LEMMA 4.1. Let $F(t)$ be an $\mathbb{R}$-valued function on $\mathbb{R}$. There are constants $K_{1}$, $K_{2}$ and $K_{3}$ such that

$$
|C(\xi F(t))| \leqslant K_{1} \xi^{2} F(t)^{2}+K_{2}|\xi||F(t)|+K_{3}
$$

for any $\xi \in \mathbb{R}$. 
Pr o of. We have

$$
\begin{aligned}
|C(\xi F(t))| \leqslant & 2^{-1} \xi^{2}\left(A+\int_{|x|<1} x^{2} \nu(d x)\right) F(t)^{2} \\
& +|\xi||F(t)|\left(|\gamma|+2^{-1} \int_{\mathbb{R}} \min \left\{1, x^{2}\right\} \nu(d x)\right)+2 \nu(\{|x| \geqslant 1\}) .
\end{aligned}
$$

Hence the lemma is true.

LEMMA 4.2. (i) $\left\{X_{t}-t: t \in \mathbb{R}\right\}$ corresponds to a homogeneous i.s.r.m. $\left\{X(B)-\lambda(B): B \in \mathcal{B}_{\mathbb{R}}^{0}\right\}$.

(ii) Let $F(t)$ be an $\mathbb{R}$-valued function on $\mathbb{R}$. Suppose $\int_{t_{0}}^{t_{1}}|F(t)| d t<\infty$ for any $t_{0}, t_{1} \in \mathbb{R}$ with $t_{0}<t_{1}$. If $F \in \mathbf{L}(\mathbf{X})$, then $F(t)$ is locally $\left\{X_{t}-t\right\}$-integrable and

$$
\int_{B} F(t) d X_{t}-\int_{B} F(t) d t=\int_{B} F(t) d\left(X_{t}-t\right) \quad \text { a.s. }
$$

for any $B \in \mathcal{B}_{\mathbb{R}}^{0}$.

Proof. (i) Let $M$ be a homogeneous i.s.r.m. corresponding to $\left\{X_{t}-t\right\}$. Then we have

$$
M((s, t])=\left(X_{t}-t\right)-\left(X_{s}-s\right)=X((s, t])-\lambda((s, t])
$$

for $s$ and $t$ with $s<t$. Notice that $\{X(B)-\lambda(B)\}$ is a homogeneous i.s.r.m. Hence it follows from Proposition 2.1 that

$$
M(B)=X(B)-\lambda(B) \text { a.s. } \quad \text { for every } B \in \mathcal{B}_{\mathbb{R}}^{0} .
$$

(ii) It is obvious from Proposition 2.5 that $F(t)$ is locally $\left\{X_{t}-t\right\}$-integrable. If $F(t)$ is a simple function, then (4.2) holds. Hence (4.2) is true.

LEMMA 4.3. Let $f(s, t)$ be an $\mathbb{R}$-valued function of class $C^{1}$ on $\widetilde{D}$. For $D_{n}$ and $D^{n}$ of Definition $\mathrm{LI}$, we have

$$
\begin{aligned}
\int_{\partial D_{n}} f(s, t) d X_{t} & =\int_{c}^{d}\left(\int_{\left(D_{n}\right)_{t}} \frac{\partial f}{\partial s}(s, t) d s\right) d X_{t} \quad \text { a.s. } \\
\int_{\partial D^{n}} f(s, t) d X_{t} & =\int_{c}^{d}\left(\int_{\left(D^{n}\right)_{t}} \frac{\partial f}{\partial s}(s, t) d s\right) d X_{t} \quad \text { a.s. }
\end{aligned}
$$

Proof. Recall that $D_{n, j}=\left\{s:(s, t) \in D_{n}, t_{j-1}^{n}<t<t_{j}^{n}\right\}$. From the definition we see that

$$
\begin{aligned}
& \int_{\partial D_{n}} f(s, t) d X_{t}=\sum_{j=1}^{l_{n}} \int_{t_{j-1}^{n}}^{t_{j}^{n}}\left(\int_{D_{n, j}} \frac{\partial f}{\partial s}(s, t) d s\right) d X_{t} \\
& =\sum_{j=1}^{l_{n}} \int_{t_{j-1}^{n}}^{t_{j}^{n}}\left(\int_{\left(D_{n}\right)_{t}} \frac{\partial f}{\partial s}(s, t) d s\right) d X_{t}=\int_{c}^{d}\left(\int_{\left(D_{n}\right)_{t}} \frac{\partial f}{\partial s}(s, t) d s\right) d X_{t} \quad \text { a.s. }
\end{aligned}
$$


Here we used Proposition 2.4 in the last equality. In the same way as above, we can prove that (4.4) is true. The lemma has been proved.

We are now ready to prove Theorem $B .1$.

Proof of Theorem B.]. As $\int_{D_{t}} \frac{\partial f}{\partial s}(s, t) d s$ is locally bounded, it follows from Corollary 2.1 that it is $\mathbf{X}$-integrable. Denote by $D_{n}$ and $D^{n}$ the inner partition and the outer partition of $\bar{D}$ with respect to $\Delta_{n}$ (see Definition L.T). We infer from Propositions 2.3 and 2.6 that

$$
\begin{aligned}
E \exp \left[i \xi\left(\int \frac{\partial}{D} \frac{\partial f}{\partial s}(s, t) d s d X_{t}-\int_{\partial D_{n}} f(s, t) d X_{t}\right)\right] \\
=E \exp \left[i \xi \int_{c}^{d}\left(\int_{\bar{D}_{t} \backslash\left(D_{n}\right)_{t}} \frac{\partial f}{\partial s}(s, t) d s\right) d X_{t}\right] \\
=\exp \left[\int_{c}^{d} C\left(\xi \int_{\bar{D}_{t} \backslash\left(D_{n}\right)_{t}} \frac{\partial f}{\partial s}(s, t) d s\right) d t\right]
\end{aligned}
$$

Let $\epsilon>0$ and set $E_{n}:=\left\{t \in[c, d]: \lambda\left(\bar{D}_{t} \backslash\left(D_{n}\right)_{t}\right)>\epsilon\right\}$. Now we split the above integral in two:

$$
\begin{aligned}
\int_{c}^{d} C\left(\xi \int_{\bar{D}_{t} \backslash\left(D_{n}\right)_{t}} \frac{\partial f}{\partial s}(s, t) d s\right) d t & =\int_{E_{n}} C\left(\xi \int_{\bar{D}_{t} \backslash\left(D_{n}\right)_{t}} \frac{\partial f}{\partial s}(s, t) d s\right) d t \\
& +\int_{[c, d] \backslash E_{n}} C\left(\xi \int_{\bar{D}_{t} \backslash\left(D_{n}\right)_{t}} \frac{\partial f}{\partial s}(s, t) d s\right) d t \\
& \equiv I_{1}+I_{2} .
\end{aligned}
$$

Let

$$
M:=\sup _{(s, t) \in \bar{D}}\left|\frac{\partial f}{\partial s}(s, t)\right| .
$$

Here it follows from Lemma 4.1 that

$$
\begin{aligned}
\left|C\left(\xi \int_{\bar{D}_{t} \backslash\left(D_{n}\right)_{t}} \frac{\partial f}{\partial s}(s, t) d s\right)\right| \leqslant & K_{1} \xi^{2}\left(\int_{\bar{D}_{t} \backslash\left(D_{n}\right)_{t}} \frac{\partial f}{\partial s}(s, t) d s\right)^{2} \\
& +K_{2}|\xi|\left|\int_{\bar{D}_{t} \backslash\left(D_{n}\right)_{t}} \frac{\partial f}{\partial s}(s, t) d s\right|+K_{3} \\
\leqslant & K_{1} \xi^{2}(M(b-a))^{2}+K_{2}|\xi| M(b-a)+K_{3} .
\end{aligned}
$$


Notice that $\lim _{n \rightarrow \infty} \lambda\left(E_{n}\right)=0$ because

$$
\lim _{n \rightarrow \infty} \int_{c}^{d} \lambda\left(\bar{D}_{t} \backslash\left(D_{n}\right)_{t}\right) d t=0 .
$$

This implies that

$$
\lim _{n \rightarrow \infty}\left|I_{1}\right| \leqslant\left(K_{1} \xi^{2}(M(b-a))^{2}+K_{2}|\xi| M(b-a)+K_{3}\right) \lim _{n \rightarrow \infty} \lambda\left(E_{n}\right)=0 .
$$

Furthermore, we see that

$$
\left|\int_{\bar{D}_{t} \backslash\left(D_{n}\right)_{t}} \frac{\partial f}{\partial s}(s, t) d s\right| \leqslant M \lambda\left(\bar{D}_{t} \backslash\left(D_{n}\right)_{t}\right) \leqslant M \epsilon
$$

on $[c, d] \backslash E_{n}$. Let $\delta>0$. As $C(\xi)$ is continuous, it follows that

$$
\left|I_{2}\right| \leqslant \int_{[c, d] \backslash E_{n}}\left|C\left(\xi \int_{\bar{D}_{t} \backslash\left(D_{n}\right)_{t}} \frac{\partial f}{\partial s}(s, t) d s\right)\right| d t<\delta \lambda\left([c, d] \backslash E_{n}\right) \leqslant \delta(d-c)
$$

for sufficiently small $\epsilon>0$ and sufficiently large $n$. This implies $\lim _{n \rightarrow \infty} I_{2}=0$. Hence we obtain

$$
\iint_{\bar{D}} \frac{\partial f}{\partial s}(s, t) d s d X_{t}-\int_{\partial D_{n}} f(s, t) d X_{t} \rightarrow 0 \quad \text { in probability }
$$

because (4.5) goes to one as $n \rightarrow \infty$. Furthermore, we have

$$
\begin{aligned}
E \exp \left[i \xi\left(\int_{\partial D^{n}} f(s, t) d X_{t}-\iint_{\bar{D}} \frac{\partial f}{\partial s}(s, t) d s d X_{t}\right)\right] \\
=\exp \left[\int_{c}^{d} C\left(\xi \int_{\left(D^{n}\right)_{t} \backslash \bar{D}_{t}} \frac{\partial f}{\partial s}(s, t) d s\right) d t\right] .
\end{aligned}
$$

In the same way as above, we arrive at the conclusion that

$$
\int_{\partial D^{n}} f(s, t) d X_{t}-\iint_{\bar{D}} \frac{\partial f}{\partial s}(s, t) d s d X_{t} \rightarrow 0 \quad \text { in probability. }
$$

Hence the theorem has been proved.

We are now ready to prove Theorem B.2. In the proofs of Theorems 3.2 and 3.3 and Corollary B.2, we use the representation $f(z)=u(z)+i v(z)$. Here $u(z)$ and $v(z)$ are the real part and the imaginary part of $f(z)$, respectively. 
Pro of of The ore m 3.2. From Theorem 3. Dit follows that almost surely

$$
\begin{aligned}
\int_{\partial D} f(z) d z_{\mathbf{X}}= & \int_{\partial D} u(s, t) d s-\int_{\partial D} v(s, t) d X_{t} \\
& +i\left(\int_{\partial D} v(s, t) d s+\int_{\partial D} u(s, t) d X_{t}\right) \\
= & -\iint_{\bar{D}} \frac{\partial u}{\partial t}(s, t) d t d s-\iint_{\bar{D}} \frac{\partial v}{\partial s}(s, t) d s d X_{t} \\
& +i\left(-\iint_{\bar{D}} \frac{\partial v}{\partial t}(s, t) d t d s+\iint_{\bar{D}} \frac{\partial u}{\partial s}(s, t) d s d X_{t}\right) \\
\equiv & I .
\end{aligned}
$$

Here we also used the usual Green's theorem. The Cauchy-Riemann equations tell us that

$$
\frac{\partial u}{\partial s}(s, t)=\frac{\partial v}{\partial t}(s, t) \quad \text { and } \quad \frac{\partial u}{\partial t}(s, t)=-\frac{\partial v}{\partial s}(s, t) .
$$

These mean that

$$
\begin{aligned}
I= & -\iint_{\bar{D}} \frac{\partial u}{\partial t}(s, t) d s d t-i \iint_{\bar{D}} \frac{\partial v}{\partial t}(s, t) d s d t \\
& +i\left(i \iint_{\bar{D}} \frac{\partial v}{\partial s}(s, t) d s d X_{t}+\iint_{\bar{D}} \frac{\partial u}{\partial s}(s, t) d s d X_{t}\right) \\
= & -i \iint_{\bar{D}} \frac{\partial f}{\partial s}(s, t) d s d t+i \iint_{\bar{D}} \frac{\partial f}{\partial s}(s, t) d s d X_{t} \\
= & i \iint_{\bar{D}} \frac{\partial f}{\partial s}(s, t) d s d\left(X_{t}-t\right) \quad \text { a.s. }
\end{aligned}
$$

The last equality is due to Lemma 4.2. The theorem has been proved.

We are now ready to prove Theorem 3.3 .

Proof of Theorem 3.3 . Let $a_{j}=\left(a_{j}^{1}, a_{j}^{2}\right) \in \mathbb{R}^{2}$ for $1 \leqslant j \leqslant n$. Arrange $\left\{a_{j}^{2}: j=1,2, \ldots, n\right\}$ in ascending order. They are denoted by

$$
\beta_{1}<\beta_{2}<\ldots<\beta_{l} \quad(l \leqslant n) .
$$

Furthermore, for each $\beta_{k}$, we arrange $\left\{a_{j}^{1}: a_{j}^{2}=\beta_{k}, 1 \leqslant j \leqslant n\right\}$ in ascending order. They are denoted by

$$
\alpha_{1}^{k}<\alpha_{2}^{k}<\ldots<\alpha_{m_{k}}^{k} .
$$


Here $\sum_{k=1}^{l} m_{k}=n$. We take a sequence $\left\{\delta_{n}\right\}$ satisfying $\delta_{n} \downarrow 0$ as $n \rightarrow \infty$. Let $\widetilde{X}_{t}=X_{t}-t$ and denote by $\widetilde{C}(z)$ the cumulant function of the distribution of $X_{1}-1$. From Lemma 4.2 and Cauchy's theorem we see that

$$
\begin{aligned}
\int_{\partial D_{\epsilon, \delta_{n}}} f(z) d z_{\mathbf{X}} & =\int_{\partial D_{\epsilon, \delta_{n}}} f(z) d z+i \int_{\partial D_{\epsilon, \delta_{n}}} f(s, t) d \widetilde{X}_{t} \\
& =i \int_{\partial D_{\epsilon, \delta_{n}}} f(s, t) d \widetilde{X}_{t} \quad \text { a.s. }
\end{aligned}
$$

We examine the convergence of $\int_{\partial D_{\epsilon, \delta_{n}}} f(s, t) d \widetilde{X}_{t}$. Let $n>m$. From Theorem 3.1 and Proposition $[2.3$ we infer that almost surely

$$
\begin{aligned}
\int_{\partial D_{\epsilon, \delta_{n}}} u(s, t) d \widetilde{X}_{t}-\int_{\partial D_{\epsilon, \delta_{m}}} u(s, t) d \widetilde{X}_{t} \\
\quad=\int_{c}^{d}\left(\int_{\left(\frac{D_{\epsilon, \delta_{n}}}{)_{t}}\right)} \frac{\partial u}{\partial s}(s, t) d s\right) d \widetilde{X}_{t}-\int_{c}^{d}\left(\frac{\int_{\left(D_{\epsilon, \delta_{m}}\right)_{t}}}{\partial u}(s, t) d s\right) d \widetilde{X}_{t} \\
=\sum_{k=1}^{l} \sum_{j=1}^{m_{k}} \int_{c}^{d}\left(\int_{J_{k, j}(t)} \frac{\partial u}{\partial s}(s, t) d s\right) d \widetilde{X}_{t} \equiv I,
\end{aligned}
$$

where

$$
J_{k, j}(t)= \begin{cases}{\left[\alpha_{j}^{k}-\epsilon, \alpha_{j}^{k}+\epsilon\right]} & \text { if } t \in\left[\beta_{k}-\delta_{m}, \beta_{k}-\delta_{n}\right] \cup\left[\beta_{k}+\delta_{n}, \beta_{k}+\delta_{m}\right], \\ \emptyset & \text { otherwise. }\end{cases}
$$

Furthermore, we have

$$
\begin{aligned}
E \exp & {\left[i \xi \int_{c}^{d}\left(\int_{J_{k, j}(t)} \frac{\partial u}{\partial s}(s, t) d s\right) d \widetilde{X}_{t}\right] } \\
& =\exp \left[\left(\int_{\beta_{k}-\delta_{m}}^{\beta_{k}-\delta_{n}}+\int_{\beta_{k}+\delta_{n}}^{\beta_{k}+\delta_{m}}\right) \widetilde{C}\left(\xi\left\{u\left(\alpha_{j}^{k}+\epsilon, t\right)-u\left(\alpha_{j}^{k}-\epsilon, t\right)\right\}\right) d t\right] .
\end{aligned}
$$

Notice that $u\left(\alpha_{j}^{k}+\epsilon, t\right)$ and $u\left(\alpha_{j}^{k}-\epsilon, t\right)$ are continuous in $t$ and thereby bounded on $\left[\beta_{k}-\delta_{m}, \beta_{k}-\delta_{n}\right] \cup\left[\beta_{k}+\delta_{n}, \beta_{k}+\delta_{m}\right]$. By Lemma 4.], this implies that

$$
\int_{c}^{d}\left(\int_{J_{k, j}(t)} \frac{\partial u}{\partial s}(s, t) d s\right) d \widetilde{X}_{t} \rightarrow 0 \quad \text { in probability }
$$

as $n, m \rightarrow \infty$. Hence $\mathrm{p}-\lim _{n, m \rightarrow \infty} I=0$. Even if we replace $u(s, t)$ with $v(s, t)$, $I$ goes to zero in probability. Hence $\int_{\partial D_{\epsilon, \delta}} f(s, t) d \widetilde{X}_{t}$ converges in probability as $\delta \downarrow 0$. 
Next, we show that this limit does not depend on $\epsilon$. Let $\epsilon^{\prime}>\epsilon>0$. Then we have almost surely

$$
\begin{aligned}
& \int_{\partial D_{\epsilon, \delta} u(s, t) d \widetilde{X}_{t}-\int_{\partial D_{\epsilon^{\prime}, \delta}} u(s, t) d \widetilde{X}_{t}}=\frac{\iint}{D_{\epsilon, \delta}} \frac{\partial u}{\partial s}(s, t) d s d \widetilde{X}_{t}-\frac{\iint}{D_{\epsilon^{\prime}, \delta}} \frac{\partial u}{\partial s}(s, t) d s d \widetilde{X}_{t} \\
&=\sum_{k=1}^{l} \sum_{j=1}^{m_{k}} \int_{c}^{d}\left(\int_{L_{k, j}(t)} \frac{\partial u}{\partial s}(s, t) d s\right) d \widetilde{X}_{t},
\end{aligned}
$$

where

$$
L_{k, j}(t)= \begin{cases}{\left[\alpha_{j}^{k}-\epsilon^{\prime}, \alpha_{j}^{k}-\epsilon\right] \cup\left[\alpha_{j}^{k}+\epsilon, \alpha_{j}^{k}+\epsilon^{\prime}\right]} & \text { if } t \in\left[\beta_{k}-\delta, \beta_{k}+\delta\right], \\ \emptyset & \text { otherwise. }\end{cases}
$$

As we see that

$$
\begin{aligned}
& E \exp \left[i \xi \int_{c}^{d}\left(\int_{L_{k, j}(t)} \frac{\partial u}{\partial s}(s, t) d s\right) d \widetilde{X}_{t}\right] \\
= & \exp \left[\int_{\beta_{k}-\delta}^{\beta_{k}+\delta} \widetilde{C}\left(\xi\left\{u\left(\alpha_{j}^{k}+\epsilon^{\prime}, t\right)-u\left(\alpha_{j}^{k}+\epsilon, t\right)+u\left(\alpha_{j}^{k}-\epsilon, t\right)-u\left(\alpha_{j}^{k}-\epsilon^{\prime}, t\right)\right\}\right) d t\right],
\end{aligned}
$$

it follows that

$$
\mathrm{p}-\lim _{\delta \downarrow 0} \int_{c}^{d}\left(\int_{L_{k, j}(t)} \frac{\partial u}{\partial s}(s, t) d s\right) d \widetilde{X}_{t}=0
$$

This implies that

$$
\int_{\partial D_{\epsilon, \delta}} u(s, t) d \widetilde{X}_{t}-\int_{\partial D_{\epsilon^{\prime}, \delta}} u(s, t) d \widetilde{X}_{t} \rightarrow 0 \quad \text { in probability }
$$

as $\delta \downarrow 0$. This convergence also holds for $v$ in place of $u$. Hence we conclude that $\mathrm{p}-\lim _{\delta \downarrow 0} \int_{\partial D_{\epsilon, \delta}} f(s, t) d \widetilde{X}_{t}$ does not depend on $\epsilon$.

The rest is to examine $\int_{\partial I_{\epsilon, \delta}\left(a_{j}\right)} f(s, t) d \widetilde{X}_{t}$ :

$$
\begin{aligned}
E \exp \left[i \xi \int_{\partial I_{\epsilon, \delta}\left(a_{j}\right)} u(s, t) d \widetilde{X}_{t}\right] & =E \exp \left[i \xi \iint_{I_{\epsilon, \delta}\left(a_{j}\right)} \frac{\partial u}{\partial s}(s, t) d s d \widetilde{X}_{t}\right] \\
& =E \exp \left[i \xi \int_{a_{j}^{2}-\delta}^{a_{j}^{2}+\delta}\left\{u\left(a_{j}^{1}+\epsilon, t\right)-u\left(a_{j}^{1}-\epsilon, t\right)\right\} d \widetilde{X}_{t}\right] \\
& =\exp \left[\int_{a_{j}^{2}-\delta}^{a_{j}^{2}+\delta} \widetilde{C}\left(\xi\left\{u\left(a_{j}^{1}+\epsilon, t\right)-u\left(a_{j}^{1}-\epsilon, t\right)\right\}\right) d t\right] .
\end{aligned}
$$


This converges to one as $\delta \downarrow 0$. Hence $\mathrm{p}-\lim _{\delta \downarrow 0} \int_{\partial I_{\epsilon, \delta}\left(a_{j}\right)} u(s, t) d \widetilde{X}_{t}=0$. This limit holds for $v$ in place of $u$ and thereby

$$
\int_{\partial D} f(s, t) d \widetilde{X}_{t}=\mathrm{p}-\lim _{\delta \downarrow 0} \int_{\partial D_{\epsilon, \delta}} f(s, t) d \widetilde{X}_{t} \quad \text { a.s. }
$$

Hence we infer from Theorems 3.$]$ and 3.2 and the usual residue formula that almost surely

$$
\begin{aligned}
\int_{\partial D} f(z) d z_{\mathbf{X}} & =\mathrm{p}-\lim _{\delta \downarrow 0}\left(i \int_{\partial D_{\epsilon, \delta}} f(s, t) d \widetilde{X}_{t}+\sum_{j=1}^{n} \int_{\partial I_{\epsilon, \delta}\left(a_{j}\right)} f(z) d z\right) \\
& =i \int_{\partial D} f(s, t) d \widetilde{X}_{t}+2 \pi i \sum_{j=1}^{n} \operatorname{Res}\left(f, a_{j}\right) .
\end{aligned}
$$

The theorem has been proved.

We are now ready to prove Theorem B.4.

Pro of of The ore $\mathrm{m}$ 3.4. We have

$$
\left|\int_{\partial D} f(z) d z_{\mathbf{X}}\right| \leqslant\left|\int_{\partial D} f(s, t) d s\right|+\left|\int_{\partial D} f(s, t) d X_{t}\right| .
$$

Let

$$
M:=\max \left\{\sup _{(s, t) \in \bar{D}}\left|\frac{\partial f}{\partial s}(s, t)\right|, \sup _{(s, t) \in \bar{D}}\left|\frac{\partial f}{\partial t}(s, t)\right|\right\} .
$$

Firstly, we see that

$$
\left|\int_{\partial D} f(s, t) d s\right| \leqslant \iint_{\bar{D}}\left|\frac{\partial f}{\partial t}(s, t)\right| d t d s \leqslant M \iint_{\bar{D}} d t d s<\infty .
$$

By Proposition 2.6 we have

$$
E \exp \left[i \xi \int_{\partial D} f(s, t) d X_{t}\right]=\exp \left[\int_{c}^{d} C\left(\xi \int_{D_{t}} \frac{\partial f}{\partial s}(s, t) d s\right) d t\right]
$$

and its Lévy measure is given by

$$
\nu_{0}(B)=\int_{c}^{d} d t \int_{\mathbb{R}} 1_{B}\left(x \int_{D_{t}} \frac{\partial f}{\partial s}(s, t) d s\right) \nu(d x) \quad \text { for any Borel set } B .
$$

By Theorem 25.3 of [15], $E\left|X_{1}\right|<\infty$ if and only if $\int_{|x|>1}|x| \nu(d x)<\infty$. Now we have

$$
\begin{gathered}
\int_{|x|>1}|x| \nu_{0}(d x)=\int_{c}^{d} d t \underset{|A(x, t)|>1}{\int_{D_{t}}}\left|x \int_{\int^{\prime}} \frac{\partial f}{\partial s}(s, t) d s\right| \nu(d x) \\
\leqslant M \int_{D} d s d t \quad \int_{|x|>(M(b-a))^{-1}}|x| \nu(d x)<\infty, \quad \text { where } A(x, t)=x \int_{D_{t}} \frac{\partial f}{\partial s}(s, t) d s .
\end{gathered}
$$


From Theorem 25.3 of [15] again it follows that $E\left|\int_{\partial D} f(s, t) d X_{t}\right|<\infty$, and thereby $E\left|\int_{\partial D} f(z) d z_{\mathbf{X}}\right|<\infty$.

Differentiating both sides of (4.6) in $\xi$ and letting $\xi=0$, we obtain the following mean:

$$
E\left[\int_{\partial D} f(s, t) d X_{t}\right]=\int_{c}^{d} \int_{D_{t}} \frac{\partial f}{\partial s}(s, t) d s d t \cdot E X_{1}=\int_{\partial D} f(s, t) d t .
$$

This yields

$$
\begin{aligned}
E\left[\int_{\partial D} f(z) d z \mathbf{X}\right] & =\int_{\partial D} f(s, t) d s+i E\left[\int_{\partial D} f(s, t) d X_{t}\right] \\
& =\int_{\partial D} f(s, t) d s+i \int_{\partial D} f(s, t) d t=\int_{\partial D} f(z) d z .
\end{aligned}
$$

The theorem has been proved.

We immediately obtain the corollary of Theorem 3.4.

Pro of of Corollary 3.2. From Theorem 3.4 we see that

$$
\begin{aligned}
E\left[\int_{\partial \triangle} f(z) d z_{\mathbf{X}}\right] & =E\left[\int_{\partial \triangle} u(z) d z_{\mathbf{X}}\right]+i E\left[\int_{\partial \triangle} v(z) d z_{\mathbf{X}}\right] \\
& =\int_{\partial \triangle} u(z) d z+i \int_{\partial \triangle} v(z) d z=\int_{\partial \triangle} f(z) d z .
\end{aligned}
$$

The usual Cauchy theorem and Morera's theorem tell us that the assertion is true.

Acknowledgments. The author would like to thank Ken-iti Sato for his valuable comments. The comments led this paper to be improved. He is also grateful to an anonymous referee for bringing to his attention the papers of A. Prékopa.

\section{REFERENCES}

[1] T. Aoyama and M. Maejima, Charcterizations of subclasses of type $G$ distributions on $\mathbb{R}^{d}$ by stochastic integral representations, Bernoulli 13 (2007), pp.148-160.

[2] O. E. Barndorff-Nielsen, M. Maejima, and K. Sato, Some classes of multivariate infinitely divisible distributions admitting stochastic integral representations, Bernoulli 12 (1) (2006), pp. 1-33.

[3] P. Billingsley, Probability and Measure, Wiley, New York-Chichester-Brisbane 1979.

[4] S. Kwapień and W. A. Woyczyński, Random Series and Stochastic Integrals: Single and Multiple, Birkhäuser, Boston, MA, 1992.

[5] M. Maejima, V. Pérez-Abreu, and K. Sato, A class of multivariate infinitely divisible distributions related to arcsine density, Bernoulli 18 (2) (2012), pp. 476-495.

[6] M. Maejima and K. Sato, Semi-Lévy processes, semi-selfsimilar additive processes, and semi-stationary Ornstein-Uhlenbeck type processes, J. Math. Kyoto Univ. 43 (3) (2003), pp. 609-639. 
[7] M. Maejima and K. Sato, The limits of nested subclasses of several classes of infinitely divisible distributions are identical with the closure of the class of stable distributions, Probab. Theory Related Fields 145 (1-2) (2009), pp. 119-142.

[8] M. Maejima and Y. Ueda, Stochastic integral characterizations of semi-selfdecomposable distributions and related Ornstein-Uhlenbeck type processes, Commun. Stoch. Anal. 3 (3) (2009), pp. 349-367.

[9] A. Prékopa, On stochastic set functions. I, Acta Math. Acad. Sci. Hungar. 7 (1956), pp. $215-$ 263.

[10] A. Prékopa, On stochastic set functions. II, Acta Math. Acad. Sci. Hungar. 8 (1957), pp. 337-374.

[11] A. Prékopa, On stochastic set functions. III, Acta Math. Acad. Sci. Hungar. 8 (1957), pp. $375-400$.

[12] B. S. Rajput and J. Rosiński, Spectral representations of infinitely divisible processes, Probab. Theory Related Fields 82 (3) (1989), pp. 451-487.

[13] A. Rocha-Arteaga and K. Sato, Topics in Infinitely Divisible Distributions and Lévy Processes, Sociedad Matemática Mexicana, México 2003.

[14] G. Samorodnitsky and M. S. Taqqu, Stable Non-Gaussian Random Processes: Stochastic Models with Infinite Variance, Chapman, New York 1994.

[15] K. Sato, Lévy Processes and Infinitely Divisible Distributions, Cambridge University Press, Cambridge 1999.

[16] K. Sato, Stochastic integrals in additive processes and application to semi-Lévy processes, Osaka J. Math. 41 (1) (2004), pp. 211-236.

[17] K. Sato, Additive processes and stochastic integrals, Illinois J. Math. 50 (1-4) (2006), pp. 825-851.

[18] K. Sato, Two families of improper stochastic integrals with respect to Lévy processes, ALEA Lat. Am. J. Probab. Math. Stat. 1 (2006), pp. 47-87.

[19] K. Sato, Monotonicity and non-monotonicity of domains of stochastic integral operators, Probab. Math. Statist. 26 (1) (2006), pp. 23-39.

[20] K. Sato, Transformations of infinitely divisible distributions via improper stochastic integrals, ALEA Lat. Am. J. Probab. Math. Stat. 3 (2007), pp.67-110.

[21] K. Sato, Description of limits of ranges of iterations of stochastic integral mappings of infinitely divisible distributions, ALEA Lat. Am. J. Probab. Math. Stat. 8 (2011), pp. 1-17.

[22] K. Sato, Inversions of infinitely divisible distributions and conjugates of stochastic integral mappings, J. Theoret. Probab. 26 (4) (2013), pp. 901-931.

[23] K. Sato, Stochastic integrals with respect to Lévy processes and infinitely divisible distributions, Sugaku Expositions 27 (1) (2014), pp. 19-42.

[24] K. Urbanik and W. A. Woyczyński, A random integral and Orlicz spaces, Bull. Acad. Polon. Sci. Sér. Sci. Math. Astronom. Phys.15 (1967), pp. 161-169.

Kouji Yamamuro

Faculty of Engineering

Gifu University

Gifu 501-1193, Japan

E-mail: yamamuro@gifu-u.ac.jp

Received on 8.12.2015;

revised version on 15.1.2018 